\title{
Genetics of Actinorhodin Biosynthesis by Streptomyces coelicolor A3(2)
}

\author{
By BRIAN A. M. RUDD* AND DAVID A. HOPWOOD \\ John Innes Institute, Colney Lane, Norwich NR4 7UH
}

(Received 27 February 1979)

\begin{abstract}
A series of 76 mutants of Streptomyces coelicolor A3(2) specifically blocked in the synthesis of the binaphthoquinone antibiotic actinorhodin were classified into seven phenotypic classes on the basis of antibiotic activity, accumulation of pigmented precursors or shunt products of actinorhodin biosynthesis, and cosynthesis of actinorhodin in pairwise combinations of mutants. The polarity of cosynthetic reactions, and other phenotypic properties, allowed six of the mutant classes to be arranged in the most probable linear sequence of biosynthetic blocks. One member of each mutant class was mapped unambiguously to the chromosomal linkage map in the short segment between the hisD and guaA loci, suggesting that structural genes for actinorhodin biosynthesis may form an uninterrupted cluster of chromosomal genes.
\end{abstract}

\section{INTRODUCTION}

Streptomyces coelicolor A3(2) produces two characterized antibiotics: methylenomycin A, the genes for the synthesis of which appear to be carried by the SCP1 plasmid (Kirby et al., 1975; Wright \& Hopwood, 1976a; Kirby \& Hopwood, 1977; Hopwood, 1978); and the pigmented antibiotic actinorhodin (Wright \& Hopwood, 1976b).

Actinorhodin is an acid-base indicator; it is blue and very soluble in polar solvents at pH values above 7 and red and poorly soluble below pH 7. Its structure (Fig. 1) was elucidated by Brockmann et al. (1966). Actinorhodin is now considered to be a member of a chemically related group of antibiotics called isochromanequinones (Zeeck et al., 1974), which also includes kalafungin (Hoeksema \& Krueger, 1976), nanaomycins (Tanaka et al., 1975; Ōmura et al., 1976), the griseusins (Tsuji et al., 1976), granaticin (Carbaz et al., 1957) and the naphthocyclinones (Zeeck \& Mardin, 1974; Zeeck et al., 1974).

In a preliminary study of the genetic determination of actinorhodin biosynthesis, five mutations blocking its production (act) were mapped to the $S$. coelicolor chromosome (Wright \& Hopwood, 1976b). Considering actinorhodin as a representative member of the isochromanequinones - and indeed of the very large and important class of polyketide streptomycete antibiotics, all of which are made by strains in which there is little or no genetic analysis (Hopwood \& Merrick, 1977) - a more detailed study of its genetic determination was undertaken. We here report the isolation and properties of 75 act mutants which fall into a minimum of seven distinct phenotypic classes. At least one member of each class was mapped to the same short segment of the chromosome, suggesting that most, if not all, of the genes specifically involved in actinorhodin biosynthesis are closely linked.

* Present address: Department of Medicinal Chemistry and Pharmacognosy, School of Pharmacy and Pharmacal Sciences, Purdue University, West Lafayette, Indiana 47907, U.S.A. 
<smiles>CCOC(=O)C[C@H]1CC2=C(C(=O)c3c(O)c(O)cc(c3O)C2)[C@@H](C)O1</smiles>

Fig. 1. The actinorhodin molecule according to Zeeck et al. (1974).

Table 1. Derivatives of Streptomyces coelicolor A3(2)

All strains were from the John Innes Institute culture collection.

Strain no.
1190
$2377^{*}$
B1 $\dagger$
B17 $\dagger$
B18 $\dagger$
B22 $\dagger$
B40 $\dagger$
B41 $\dagger$
B201
B204
B210
B211
B212
B241
B400
2808

Genetic markers

Plasmid status

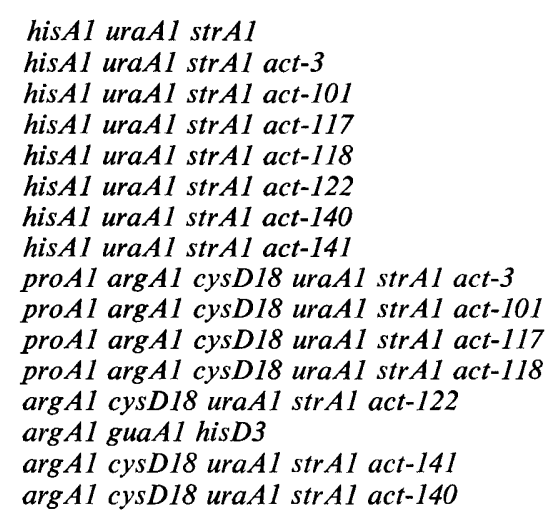

$\mathrm{SCP}^{-}{ }^{-} \mathrm{SCP} 2^{+}$

SCP1- SCP2 ${ }^{+}$

$\mathrm{SCP} 1-\mathrm{SCP} 2^{+}$

SCP1- ${ }^{-} \mathrm{SP} 2^{+}$

SCP1 ${ }^{-} \mathrm{SCP}^{+}$

SCP1- $\mathrm{SCP} 2^{+}$

SCP1- SCP2 ${ }^{+}$

SCP1- SCP2 ${ }^{+}$

SCP1- SCP2*

SCP1- SCP2*

SCP1- SCP2*

SCP1- SCP2*

SCP1- SCP2*

SCP1- SCP2*

SCP1- SCP2*

SCP1- ${ }^{-} \mathrm{SCP} 2 *$

* An act mutant, derived from strain 1190 and described by Wright \& Hopwood (1976b), adopted during the course of this work as the type strain of act class II.

$\dagger$ Mutant derivatives of strain 1190 isolated in the course of this work.

\section{METHODS}

General. Complete medium (CM), minimal medium (MM) and general cultural and genetic techniques were those described by Hopwood (1967). Nutrient agar was Oxoid nutrient agar (ONA). For u.v.mutagenesis, spore suspensions were irradiated at a dose rate of approximately $4.4 \mathrm{~W} \mathrm{~m}^{2}$ for $160 \mathrm{~s}$, giving a survival of about $1 \%$.

Strains. Streptomyces coelicolor strains (Table 1) were mutational and recombinational derivatives of strain A3(2) (Hopwood et al., 1973). This is a strain of Streptomyces violaceoruber according to Kutzner \& Waksman (1959). The locations on the circular linkage map of the markers used in genetic analysis are shown in Fig. 2. Micro-organisms used in testing for sensitivity to actinorhodin or related compounds were those listed by Wright \& Hopwood (1976a).

Isolation of act mutants. The parent strain was that chosen by Wright \& Hopwood (1976b), the SCP1strain 1190. Irradiated spores of many separate clones were plated to yield 500 to 1000 colonies per plate on CM. On this medium, actinorhodin accumulates in the mycelium in the red acidic form (proto-actinorhodin) which is relatively insoluble in water; thus act mutants are not obscured by nearby act $t^{+}$colonies even on crowded plates. Exposure of mature colonies to ammonia fumes, by inverting the plates over $5 \mathrm{ml}$ of $35 \%$ ammonia solution in a Petri dish lid, caused conversion of proto-actinorhodin to the blue, watersoluble form; blue droplets appeared on the colony surface and haloes of blue diffusing pigment developed around the colonies. Presumptive act colonies, recognized by lack of this response after a short period (Fig. 3), were picked and purified.

Cosynthesis tests. Patches of mutants, a few millimetres apart, were made on CM plates. Cosynthesis of actinorhodin was revealed by ammonia fuming or by overlaying the plates with Staphylococcus aureus in ONA (Fig. 4).

Tests of antibacterial activity. Plugs $(7 \mathrm{~mm}$ diam.) were cut from $5 \mathrm{~d}$-old confluent $\mathrm{CM}$ plate cultures of act mutants and other strains, embedded in ONA seeded with the indicator organism, and incubated overnight at the optimal growth temperature of the indicator. 


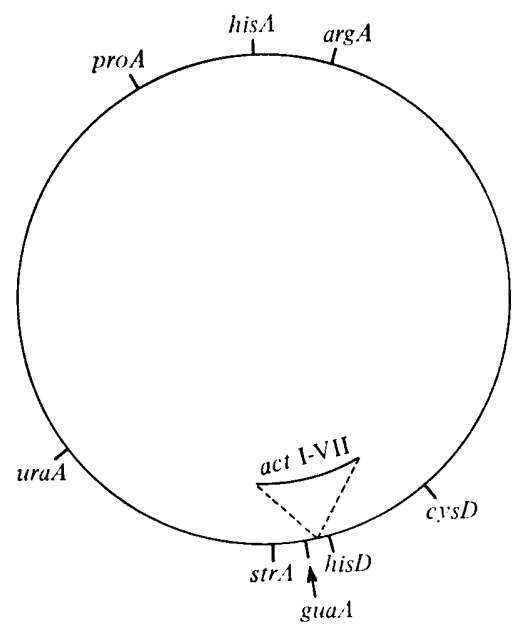

Fig. 2. Linkage map of S. coelicolor A3(2) showing locations of the markers referred to in this paper.

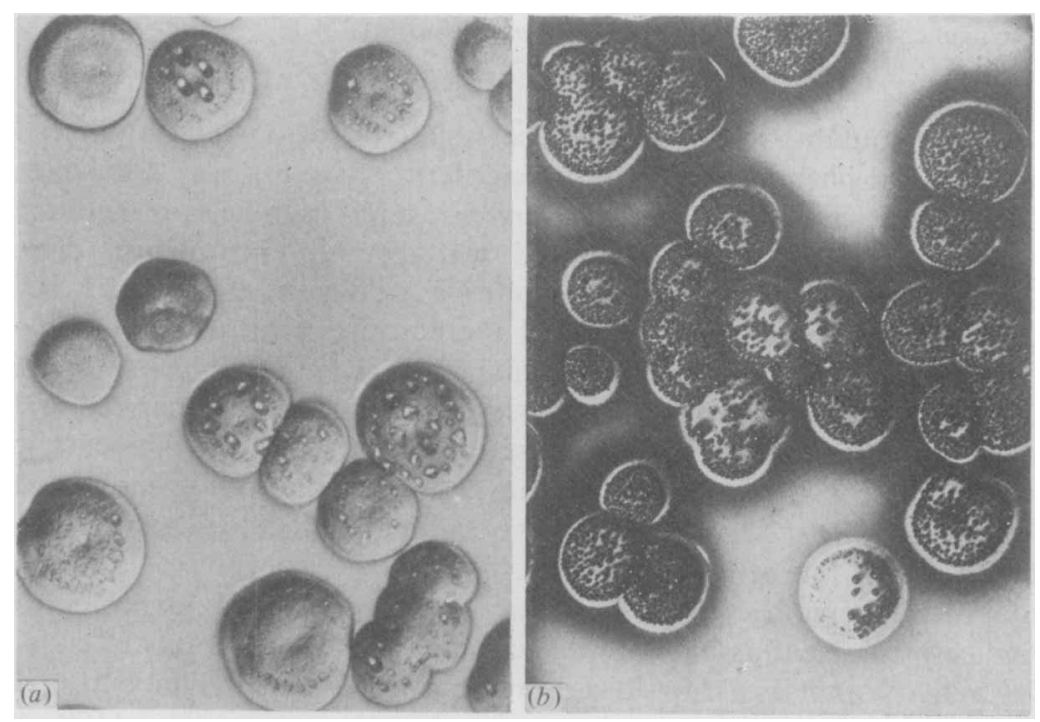

Fig. 3. Isolation of act mutants. Colonies of strain 1190 grown on CM agar for $5 \mathrm{~d}(a)$ were exposed to ammonia fumes $(b)$. Presumptive act mutants (one is seen at the bottom of $b$ ) showed no blue droplets or the halo of blue diffusing pigment characteristic of the starting strain.

\section{RESULTS}

\section{Isolation and phenotypic classification of act mutants}

Of 129 presumptive act mutants isolated, 75 were interpreted as having lesions specifically affecting actinorhodin synthesis since they appeared normal in other respects; they were 'true' act mutants. A few of these mutants produced small quantities of actinorhodin; by other phenotypic criteria (see below) they were interpreted as carrying leaky act mutations of particular classes. The remaining 54 strains were defective, to varying degrees, in differentiation, some being completely devoid of aerial mycelium (bld mutants; Merrick, 1976) and most of them produced limited quantities of actinorhodin; these strains were not studied further. The frequency of occurrence of 'true' act mutants after u.v.-irradiation was about $0 \cdot 2 \%$ (for example, 21 out of 10700 colonies in one experiment). 


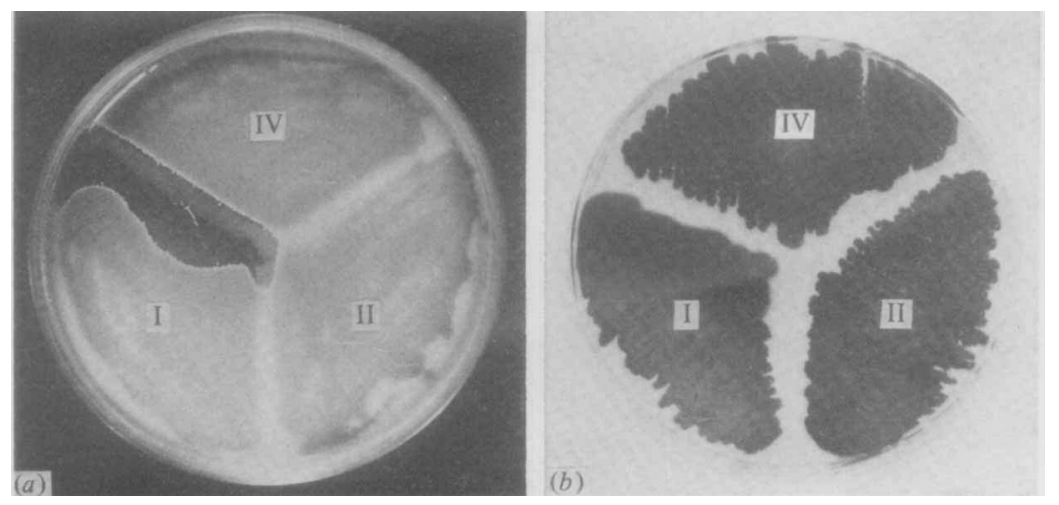

Fig. 4. Cosynthesis tests between act mutants of classes I, II and IV. Patches of three mutants were made on two CM plates, in mirror image orientation. In $(a)$, seen from above, the surface mycelial growth was killed by chloroform after $5 \mathrm{~d}$ and overlaid with Staph. aureus in nutrient agar. After overnight incubation, cosynthesis of actinorhodin is revealed by inhibition of the overlay. In (b), seen from below, cosynthesis was revealed by exposing the plate to ammonia fumes: a dark band of (blue) pigment has developed in that part of the converter class I mutant closest to the secretor class IV mutant. The class II mutant is not involved in cosynthesis. Note the corresponding location of the blue pigment $(b)$ and the inhibition of Staph. aureus $(a)$.

The 75 new act mutants and one of those isolated previously (Wright \& Hopwood, $1976 b$ ) were classified phenotypically by three criteria: colour of any diffusible pigment(s) produced; antibiotic activity against Staph. aureus; and cosynthesis of actinorhodin with other mutants. Cosynthesis, where it occurred, was always non-reciprocal, one mutant (of one class) acting as secretor to another mutant (of a different class) which acted as converter (Fig. 4). The secretor, which supplied the converter with a precursor or cofactor required for actinorhodin biosynthesis, presumably carried a later block in the biosynthetic sequence than the converter mutant. The classes of mutant are summarized in Table 2.

Members of five of the seven mutant classes produced diffusible pigments, which were apparently different for each cosynthetic class. Most mutants appeared to produce a mixture of pigments at least some of which were acid-base indicators, and resembled actinorhodin in being less soluble at low $\mathrm{pH}$ values.

Apart from the two members of class VII, which produced small quantities of a red/blue acid-base indicator pigment assumed to be actinorhodin, only mutants of class $\mathrm{V}$ had any antibacterial activity and this showed a somewhat different specificity from that of the wild-type $\left(a c t^{+}\right)$: class $\mathrm{V}$ mutants were less active than the parent strain 1190 against some Gram-positive bacteria, notably Bacillus cereus, Micrococcus lysodeikticus, Streptococcus faecalis and Corynebacterium glutamicum, but there was little or no difference between class V mutants and the wild-type in their activity against Bacillus subtilis and Staph. aureus.

The mycelium of mutants of classes I and II, which produced no diffusible pigment, contained a red, non-diffusible pigment which was apparently genetically and structurally unrelated to actinorhodin (Rudd, 1978). This pigment was also present in act ${ }^{+}$strains and in mutants of other act classes where it tended to be masked by diffusible pigments.

\section{Genetic analysis of act mutations}

Data from preliminary experiments to map a selection of 12 act mutations, in which each mutant was crossed with strains carrying a complementary set of standard markers, were consistent with a position for each act mutation between $c y s D$ and $\operatorname{str} A$ on the linkage map (Fig. 2). The mutants included the type member of each of the seven phenotypic classes 
Genetics of actinorhodin biosynthesis

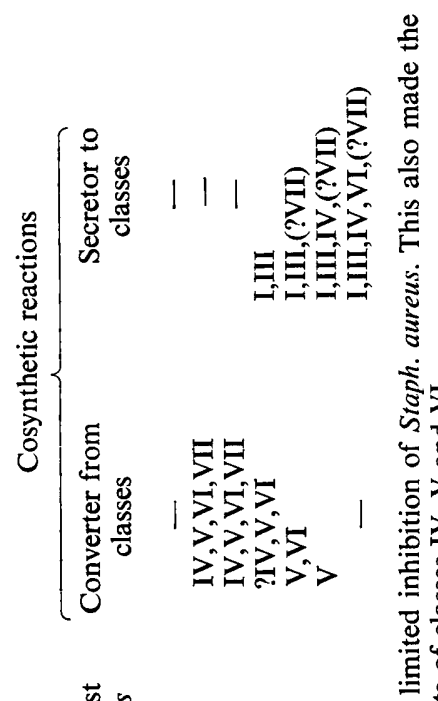

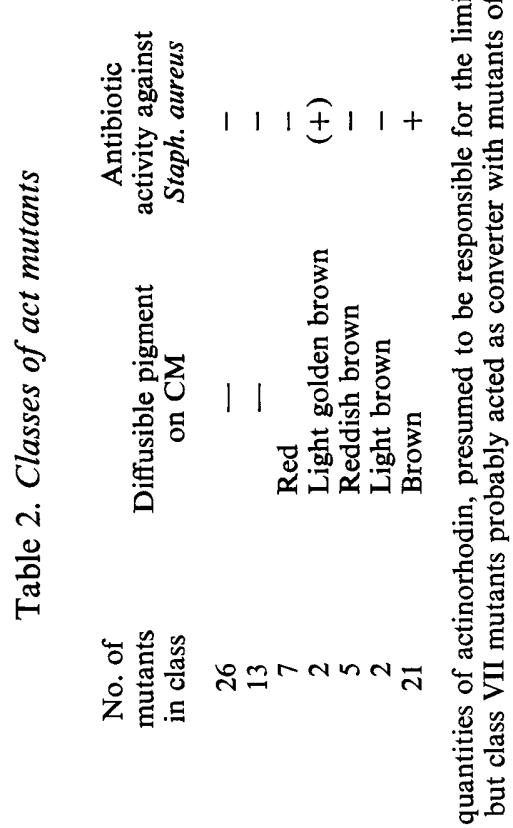

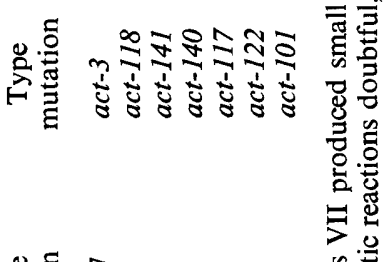

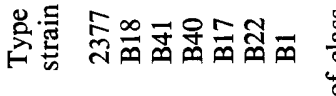

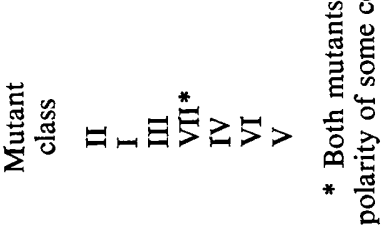




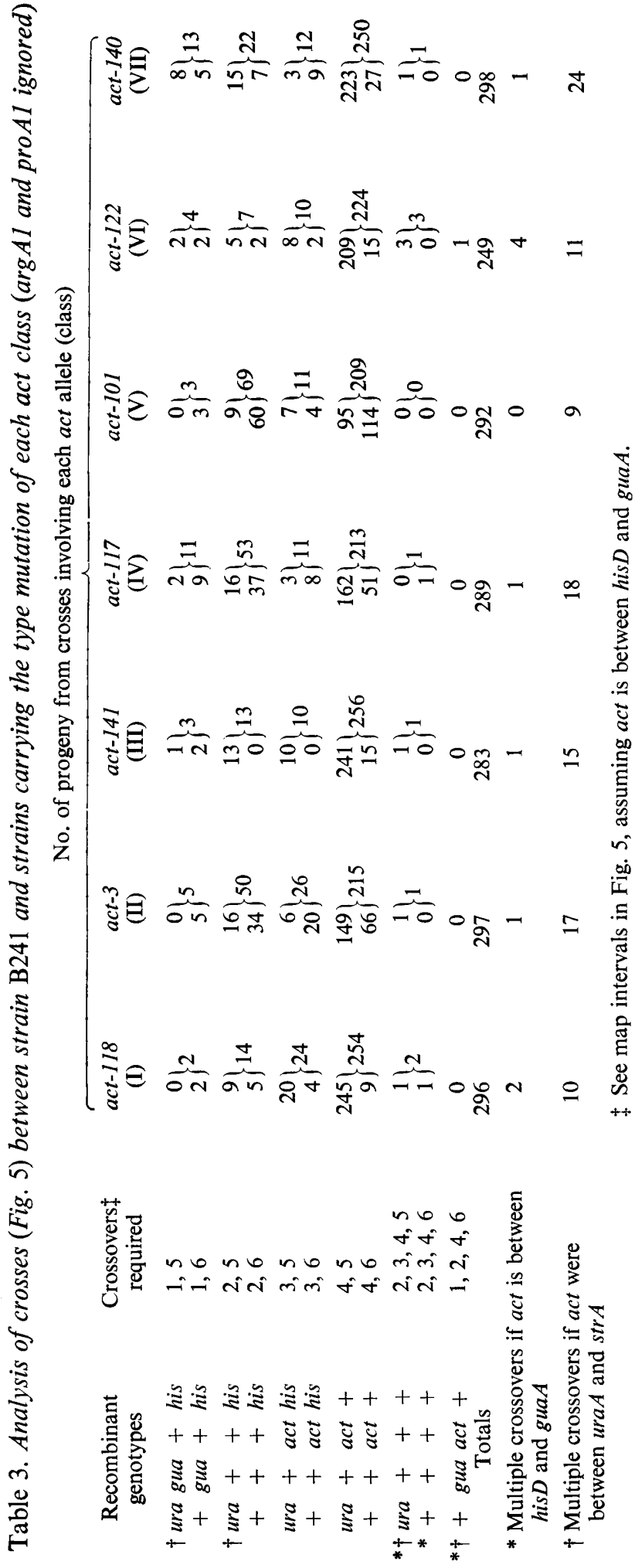




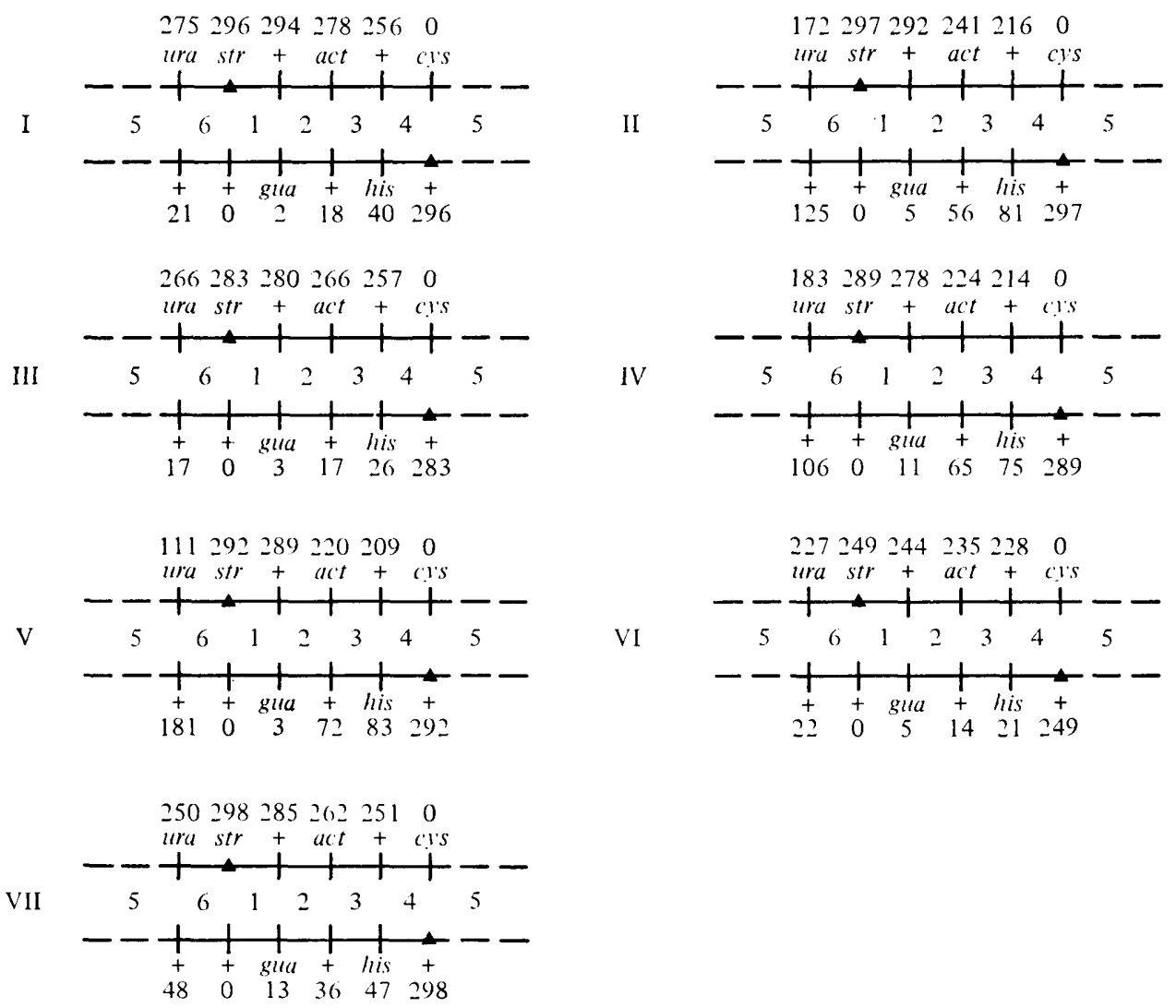

Fig. 5. Allele frequencies in crosses of strain B241 with strains carrying the type mutation of each act class, calculated from the data in Table 3. Roman numerals indicate the class of the act mutant introduced into the cross in the following parent strains: I, B211; II, B201; III, B400; IV, B210; V, B204; VI, B212; VII, 2808.

listed in Table 2, together with one additional member of each of classes I and II and three of class $\mathrm{V}$.

More precise analysis was carried out for each of the seven type mutations by making crosses between recombinant derivatives of the original mutant strains and strain B241, with selection for $\operatorname{str} A 1$ and $c y s D^{+}$. All the parents lacked the SCP1 plasmid but carried the second recognized sex factor, SCP2, in its variant form SCP2* (Bibb et al., 1977); such crosses are convenient for genetic analysis since they have an enhanced recombination frequency compared with $\mathrm{SCP} 2+\times \mathrm{SCP} 2^{+}$crosses and are typically non-polarized, each parent apparently functioning as donor or recipient with about equal probability (Bibb, 1978). In each cross, the allele ratio for $a c t^{+} / a c t$ was consistent with a map location either between $u r a A$ and str $A$ or, as indicated by the preliminary crosses, between $c y s D$ and str $A$. The former location was excluded by the data, since it required relatively large proportions of recombinants to have arisen by multiple crossing-over (Table 3). The position of each act mutation in the cys $D$-str $A$ segment was therefore narrowed down to the short interval between his $D$ and guaA since only this location was compatible with the gradient of allele frequencies at the hisD, act and guaA loci (Fig. 5). 


\section{DISCUSSION}

Mutations leading specifically to non-production of actinorhodin fell into seven distinct phenotypic classes. Two of the classes each contained only two members so the possibility exists that other classes of mutants remain to be isolated. The cosynthetic reactions of most of the mutant classes are compatible with sequential blocks in a linear biosynthetic sequence:

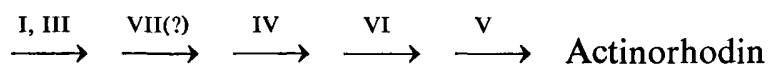

Since their cosynthetic reactions were identical, the relative order of the blocks in mutants of classes I and III cannot be determined; however, since class III mutants secreted a diffusible pigment, they are perhaps more likely to represent the later block. The position of class VII mutants in the sequence is somewhat equivocal since both the mutants of this class were leaky; production of small quantities of actinorhodin by these mutants cast some doubt on reactions in which class VII mutants appeared to act as converter. However, their converter reaction with class IV mutants, at least, was reasonably certain, serving to establish their position in the sequence. Interestingly, class V mutants, which were the only mutants to have antibiotic activity against Staph. aureus not attributable to leaky actinorhodin production, represent blocks in the final step in the sequence defined by this set of mutants. Perhaps an immediate precursor of actinorhodin, or a shunt product of it, is antibiotically active.

Class II mutants, which showed no cosynthesis with any other mutant, cannot be included in the biosynthetic sequence. Regulatory mutations may be included in this class, but its large size ( 26 out of the 76 mutants) may make it unlikely that all members of the class are of this type. Another possibility is that this class includes polar mutations within an operon.

It is possible that mutants in classes IV to VII, which can act alternatively as secretor or converter, carry defects in genes determining single function enzymes involved in modifications of the molecule subsequent to the formation of the basic structure from a polyketide chain, while mutations in classes I and III, which act only as converter, are more likely to represent defects in a multifunctional aromatic synthetase which may assemble the chain. That the polyketide route (Birch, 1967) is indeed involved in actinorhodin biosynthesis, as is likely $a$ priori, is strongly suggested by the finding that the nanaomycins, which closely resemble the actinorhodin half-molecule, are appropriately labelled by $\left[1-{ }^{13} \mathrm{C}\right]$ acetate (Tanaka et al., 1975). ${ }^{13} \mathrm{C}$-labelling experiments with the related compounds granaticin (Snipes et al., 1979) and $\alpha$-naphthocyclinone (Schröder \& Floss, 1978) also support this mode of derivation of the basic isochromane naphthoquinone unit.

At least one mutation of each of the seven classes of act mutations was mapped between the closely linked chromosomal loci his $D$ and guaA, suggesting that the genes directly involved in actinorhodin biosynthesis may form an uninterrupted cluster on the chromosome. This raises the interesting possibility that an operon may be involved. Recent developments in the study of plasmids in S. coelicolor (Bibb et al., 1977) and of conditions for the efficient transformation of protoplasts by plasmid DNA (Bibb et al., 1978) open the possibility of gene cloning in this organism. Analysis of the physical organization of the actinorhodin biosynthetic genes and of their regulation by in vitro studies are therefore exciting possibilities for the future.

B. A. M. Rudd gratefully acknowledges a graduate studentship provided by Pfizer Ltd. We thank: Keith Chater for valuable discussions. 


\section{REFERENCES}

BIBB, M. J. (1978). Genetical and physical studies of a Streptomyces coelicolor plasmid. Ph.D. thesis, University of East Anglia, Norwich.

Bibb, M. J., Freeman, R. F. \& Hopwood, D. A. (1977). Physical and genetical characterisation of a second sex factor, SCP2, for Streptomyces coelicolor A3(2). Molecular and General Genetics 154, 155-166.

BIBB, M. J., WARD, J. M. \& Hopwood, D. A. (1978). Transformation of plasmid DNA into Streptomyces at high frequency. Nature, London 274, 398-400.

BIRCH, A. J. (1967). Biosynthesis of polyketides and related compounds. Science 156, 202-206.

Brockmann, H., Zeeck, A., Merwe, K. Van der \& MülleR, W. (1966). Die Konstitution des Actinorhodins. Justus Liebigs Annalen der Chemie 698, 209-229.

Carbaz, R., EtTinger, L., GäUmanN, E., Kalvoda, J., Keller-Schierlein, W., Kradolfer, F., Manukian, B. K., NeipP, L., Prelog, V., Reusser, P. \& ZÄHNER, H. (1957). StoffwechselProdukte von Actinomyceten: Granaticin. Helvetica chimica acta 40, 1262-1269.

Hoeksema, H. \& KRueger, W. C. (1976). Kalafungin. II. Chemical transformations and the absolute configuration. Journal of Antibiotics 29, 704-709.

Hopwood, D. A. (1967). Genetic analysis and genome structure in Streptomyces coelicolor. Bacteriological Reviews 31, 373-403.

Hopwood, D. A. (1978). Extrachromosomally determined antibiotic production. Annual Review of Microbiology 32, 373-392.

Hopwood, D. A. \& Merrick, M. J. (1977). Genetics of antibiotic production. Bacteriological Reviews 41, 595-635.

Hopwood, D. A., Chater, K. F., Dowding, J. E. \& ViviAN, A. (1973). Advances in Streptomyces coelicolor genetics. Bacteriological Reviews 37, 371-405.

KIRBY, R. \& Hopwood, D. A. (1977). Genetic determination of methylenomycin synthesis by the SCP1 plasmid of Streptomyces coelicolor A3(2). Journal of General Microbiology 98, 239252.

Kirby, R., Wright, L. F. \& Hopwood, D. A. (1975). Plasmid-determined antibiotic synthesis and resistance in Streptomyces coelicolor. Nature, London 254, 265-267.
Kutzner, H. J. \& WAKSMAN, S. A. (1959). Streptomyces coelicolor Müller and Streptomyces violaceoruber Waksman and Curtis, two distinctly different organisms. Journal of Bacteriology 78, 528-538.

MerRICK, M. J. (1976). A morphological and genetic mapping study of bald colony mutants of Streptomyces coelicolor. Journal of General Microbiology 96, 299-315.

Omura, S., Tanaka, H., OKada, Y. \& Marumo, H. (1976). Isolation and structure of nanaomycin $D$, an enantiomer of the antibiotic kalafungin. Journal of the Chemical Society, Chemical Communications, 320-321.

RuDD, B. A. M. (1978). Genetics of pigmented secondary metabolites in Streptomyces coelicolor. Ph.D. thesis, University of East Anglia, Norwich.

SCHRöDER, K. \& Floss, H. G. (1978). Biosynthesis of $\alpha$-naphthocyclinone. Journal of Organic Chemistry 43, 1438-1441.

Snipes, C. E., Chang, C. \& Floss, H. G. (1979). Biosynthesis of the antibiotic granaticin. Journal of the American Chemical Society 101, 701-706.

Tanaka, H., Koyama, Y., Nagal, T., Marumo, H. \& ŌMURA, S. (1975). Nanaomycins, new antibiotics produced by a strain of Streptomyces. II. Structure and biosynthesis. Journal of Antibiotics 28, 868-875.

Tsuji, N., Kobayashi, M., Terui, Y. \& Tori, K. (1976). The structure of griseusins $A$ and $B$, new isochromanequinone antibiotics. Tetrahedron 32, 2207-2210.

Wright, L. F. \& Hopwood, D. A. (1976a). Identification of the antibiotic determined by the SCP1 plasmid of Streptomyces coelicolor A3(2). Journal of General Microbiology 95, 96-106.

WRIGHT, L. F. \& Hopwood, D. A. (1976b). Actinorhodin is a chromosomally determined antibiotic in Streptomyces coelicolor A3(2). Journal of General Microbiology 96, 289-297.

ZEECK, A. \& MARDIN, M. (1974). Isolierung und Konstitution von $\alpha$-Naphthocyclinon. Justus Liebigs Annalen der Chemie, 1063-1099.

ZEECK, A., ZÄHNER, H. \& MARDIN, M. (1974). Isolierung und Konstitution der Isochromanchinon-Antibiotica $\beta$ und $\gamma$-Naphthocyclinon. Justus Liebigs Annalen der Chemie, 1100-1125. 